\section{Anakinra and Its Rapidly Expanding Role in Management of Nonarthritic Systemic Disorders}

\section{To the Editor:}

The recent article by Le Loët, et al was highly interesting ${ }^{1}$. The authors have shown that anakinra in combination with disease modifying antirheumatic drug (DMARD) therapy safely improved functional status in patients with active rheumatoid arthritis (RA). Interestingly, while anakinra is used primarily for management of rheumatological disorders, it is increasingly used for management of other nonarthritic systemic diseases. Recent studies reveal that anakinra has an important role in management of cardiologic diseases. It decreases necrosis of cardiac tissue following acute myocardial infarctions ${ }^{2}$, and thereby attenuates cardiac remodeling and prevents apoptosis and subsequent postinfarct complications such as cardiac failure. In patients with RA, anakinra increases the coronary flow reserve and decreases endothelin-1 levels, improving left ventricular function in these patients ${ }^{3}$. Similarly, Chada, et al have shown in an animal model that when anakinra is administered as an aerosol to surfactantdepleted lungs it reduces pulmonary inflammation, but also has a negative effect on the pulmonary artery pressure and thus ameliorates associated pulmonary hypertension ${ }^{4}$.

Besides providing these cardiothoracic benefits, anakinra is also useful for management of other systemic diseases. It is highly effective in attenuating the symptoms such as fever and rash associated with tumor necrosis factor receptor-associated periodic syndrome (TRAPS) ${ }^{5}$. Indeed, it prevents relapses when used in patients with TRAPS. It also has a role in the management of neurologic diseases - it has been shown to ameliorate sensory deafness in patients with Muckle-Wells syndrome ${ }^{6}$. Anakinra also decreases hyperalgesia associated with conditions such as osteosarcomas by inhibiting interleukin 1 (IL-1) ${ }^{7}$. Anakinra also seems to be an emerging and promising alternative for management of endocrine disorders. Sauter, et al have shown that IL-1 receptor antagonists induce $B$-cell proliferation and prevent $\beta$-cell apoptosis, preventing the development of diabetes ${ }^{8}$.

Clearly, anakinra exerts beneficial effects in multiple different systemic diseases. There is an urgent need for further studies to confirm these findings and to identify other systemic diseases in which it may play a beneficial role.

SHAILENDRA KAPOOR, MD. Address reprint requests to Dr. S. Kapoor, 413-75 Kristin Circle, Schaumburg, Illinois 60195. E-mail: shailendrakapoor@yahoo.com

\section{REFERENCES}

1. Le Loet X, Nordstrom D, Rodriguez M, et al. Effect of anakinra on functional status in patients with active rheumatoid arthritis receiving concomitant therapy with traditional disease modifying antirheumatic drugs: Evidence from the OMEGA trial. J Rheumatol 2008;35:1538-44.

2. Abbate A, Salloum FN, Vecile E, et al. Anakinra, a recombinant human interleukin-1 receptor antagonist, inhibits apoptosis in experimental acute myocardial infarction. Circulation 2008;117:2670-83.

3. Ikonomidis I, Lekakis JP, Nikolaou M, et al. Inhibition of interleukin-1 by anakinra improves vascular and left ventricular function in patients with rheumatoid arthritis. Circulation 2008;117:2662-9.

4. Chada M, Nögel S, Schmidt AM, et al. Anakinra (IL-1R antagonist) lowers pulmonary artery pressure in a neonatal surfactant depleted piglet model. Pediatr Pulmonol 2008;43:851-7.

5. Gattorno M, Pelagatti MA, Meini A, et al. Persistent efficacy of anakinra in patients with tumor necrosis factor receptor-associated periodic syndrome. Arthritis Rheum 2008;58:1516-20.

6. Yamazaki T, Masumoto J, Agematsu K, et al. Anakinra improves sensory deafness in a Japanese patient with Muckle-Wells syndrome, possibly by inhibiting the cryopyrin inflammasome. Arthritis Rheum 2008;58:864-8.

7. Baamonde A, Curto-Reyes V, Juarez L, Meana A, Hidalgo A, Menendez L. Antihyperalgesic effects induced by the IL-1 receptor antagonist anakinra and increased IL-1ß levels in inflamed and osteosarcoma-bearing mice. Life Sci 2007;81:673-82.

8. Sauter NS, Schulthess FT, Galasso R, Castellani LW, Maedler K. The antiinflammatory cytokine interleukin-1 receptor antagonist protects from high-fat diet-induced hyperglycemia. Endocrinology 2008;149:2208-18.

J Rheumatol 2009;36:2; doi:10.3899/jrheum.080802 